\title{
Co-Ti- and Mn-Ti-substituted barium ferrite for electromagnetic property tuning and enhanced microwave absorption synthesized via mechanical alloying
}

\begin{abstract}
Substituted barium hexaferrite nanoparticles with nominal composition of BaCo1.0Ti1.0Fe10O19 and BaMn0.8Ti0.8Fe10.4O19 were synthesized by high energy ball milling (HEBM). The effects of $\mathrm{Co}-\mathrm{Ti}$ and $\mathrm{Mn}-\mathrm{Ti}$ substitution on their microstructure, electromagnetic properties, and microwave absorptive behavior were analyzed. The samples were structurally characterized by X-ray diffractometry (XRD), field emission scanning electron microscopy (FESEM), and energy-dispersive X-ray analysis (EDX). The M-H loops of the composites were determined with a vibrating sample magnetometer (VSM), and the interaction with the microwave radiation in the range of $8-18 \mathrm{GHz}$ of the nanocomposites dispersed in epoxy resin was measured with a vector network analyzer (VNA). This study suggests that by controlling the grain size and different elements of substitution would give a decrease in coercivity and enhanced values of complex permittivity in order to improve microwave absorption. The dielectric constant and loss were enhanced in comparison to the permeability constant and loss over the entire frequency range. Finally, microwave measurement showed that the substituted barium hexaferrite sample with $\mathrm{Co}-\mathrm{Ti}$ and $\mathrm{Mn}-\mathrm{Ti}$ could be used as an efficient microwave absorption material with an appropriate absorption at -31.27 and $-26.73 \mathrm{~dB}$, respectively. The predicted absorption and reflection loss demonstrates that $\mathrm{Co}-\mathrm{Ti}$ and $\mathrm{Mn}-\mathrm{Ti}$ substitution gives low reflectivity at microwave frequency and is a good candidate for electromagnetic materials for radar wave applications.
\end{abstract}

Keyword: Barium hexaferrite (BaFe12O19); High-energy ball milling (HEBM); Magnetic properties; Dielectric properties 